\title{
Derivations of the Frobenius Map
}

\author{
Piotr Kowalski* \\ Wrocław University \\ Univ. Illinois at Urbana-Champaign
}

\begin{abstract}
We prove that the theory of fields with a derivation of Frobenius has the model companion which is stable and admits elimination of quantifiers up to the level of the $\lambda$-functions. Along the way, we give new geometric axioms of $\mathrm{DCF}_{p}$.
\end{abstract}

\section{Introduction}

Throughout this paper $\Omega$ is a big algebraically closed field of characteristic $p>0$, all the other fields are embedded into $\Omega, n \geq 0$ and $q=p^{n}$. Let $K$ be a field and $\delta: K \longrightarrow K$. $\delta$ is a derivation of the $n$-th power of Frobenius if for any $a, b \in K$,

$$
\delta(a+b)=\delta(a)+\delta(b), \quad \delta(a b)=b^{q} \delta(a)+a^{q} \delta(b) .
$$

Let $\mathrm{DF}_{p, n}$ denotes the theory of fields with a derivation of the $n$-th power of Frobenius. For brevity, we call a derivation of the $n$-th power of Frobenius an $n$-derivation and a field with an $n$-derivation an $n$-differential field. Similarly, we talk about $n$-differential fields extensions, etc. Thus differential fields are 0 -differential fields in our terminology. A natural example of an $n$-derivation is the composition of the $n$-th power of Frobenius with a derivation. However, not all the $n$-derivations can be obtained in such a way.

* Supported by a postdoc under NSF Focused Research Grant DMS 01-00979 and the Polish KBN grant 2 P03A 01824 
In this paper we prove that $\mathrm{DF}_{p, n}$ has the model companion $\mathrm{DCF}_{p, n}$, which behaves much as the theory of differentially closed fields in positive characteristic (see [Wo1]); in particular it is stable, not superstable and admits elimination of quantifiers in the language with the $\lambda$-functions. We do not know if adding just the $p$-th root function is enough, as in the case of $\mathrm{DCF}_{p}$.

The axioms of $\mathrm{DCF}_{p, n}$ are in the style of Pierce-Pillay axioms of $\mathrm{DCF}_{0}$ $[\mathrm{PP}]$. In particular, this gives new axioms of $\mathrm{DCF}_{p}$. The proof that $\mathrm{DCF}_{p, n}$ does axiomatize the model companion of $\mathrm{DF}_{p, n}$ follows the lines from $[\mathrm{PP}]$, however some care needs to be taken while dealing with separability issues.

The main motivation for considering $n$-derivations is a theorem [Kow, 2.3.1] saying that the $n$-derivations constitute the remaining (besides derivations and endomorphisms) class of jet operators [Bu] (up to a natural notion of equivalence of operators $[\mathrm{Bu}]$ ) on fields. Therefore, this paper shows that for any jet operator, its theory has the model companion.

At first I thought that the Shelah-Wood theory [Sh], [Wo1] can be extended smoothly to the case of $n$-derivations just by "twisting arguments by a power of Frobenius". However, not all the arguments from the differential algebra extend to the $n$-differential case. Some results trivially follow from functorial properties of the structure ring together with the existence of extensions to fields of fractions. For others some computations are needed, but there are always twisted versions of the proofs from differential algebra. For example, the role of the tangent space is played by the Frobenius tangent space. Finally, there are some results which do not hold for derivations of non-zero powers of Frobenius. Note that the real difference between derivations and $n$-derivations can be seen via looking at compositions. The composition of an $n$-derivation with an $m$-derivation is an $n+m$-derivation for $m>0$ and is not an $l$-derivation for any $l$, if $m=0$. The lack of higher order derivations is the main obstacle for proving the quantifier elimination up to the level of the (partial) inverse of Frobenius.

I would like to thank Zoé Chatzidakis and Ludomir Newelski for their comments and suggestions. I would like also to thank the referee for many valuable suggestions. 


\section{Some $n$-differential algebra}

To prove certain properties of $n$-derivations, we need to go beyond the context of fields. All the rings considered in this section are of characteristic $p$ commutative and with unity. One can associate with a ring $R$ its structure ring of $n$-derivations $R_{(1)}:=\left(R^{2}, \oplus, *\right)$, where:

$\left(a_{0}, a_{1}\right) \oplus\left(b_{0}, b_{1}\right)=\left(a_{0}+a_{1}, b_{0}+b_{1}\right),\left(a_{0}, a_{1}\right) *\left(b_{0}, b_{1}\right)=\left(a_{0} b_{0}, a_{0}^{q} b_{1}+b_{0}^{q} a_{1}\right)$.

One can check that $R_{(1)}$ is really a ring $(\mathbf{0}=(0,0), \mathbf{1}=(1,0))$. This is functorial: for $f: R \longrightarrow S$ we define $f_{(1)}: R_{(1)} \longrightarrow S_{(1)}$ as $f \times f$.

Definition 1.1 Let $R$ be a ring. $A$ map $\delta: R \rightarrow R$ is an $n$-derivation if the map $R \ni a \mapsto(a, \delta(a)) \in R_{(1)}$ is a ring homomorphism.

One easily checks that this definition coincides with the one given in the introduction. Thus $n$-derivations are jet operators in the sense of [Bu] (see also $[\mathrm{Kow}])$, or $\mathcal{D}$-operators from $[\mathrm{Sc}]$.

Since $R_{(1)}$ is a ring, we get that $n$-derivations not coming from derivations (i.e. not being the compositions of the $n$-th power of Frobenius with a derivation) exist. Consider the zero $n$-derivation on a field $K$. Since an $n$ derivation on $K[X]$ is equivalent to a homomorphism from $K[X]$ to $K[X]_{(1)}$, for any $F \in K[X], X \mapsto F$ extends uniquely to an $n$-derivation of $K[X]$. It is enough to choose $F$ not belonging to $K[X]^{p}$, to get an $n$-derivation not coming from a derivation. Using Lemma 1.4, we can produce such an $n$-derivation on a field.

If $f: R \longrightarrow S$ is a homomorphism of rings, we call a map $\delta: R \longrightarrow S$ an $n$-derivation of $f$, if $\delta$ is additive and the map $(f, \delta): R \rightarrow S_{(1)}$ is a ring homomorphism. So the usual $n$-derivations can be considered as $n$ derivations of the identity map. After Fact 1.2, $f$ is always an inclusion map, and all the $n$-derivations are denoted by $\delta$.

As was noted by Udi Hrushovski and David Pierce, $n$-derivations on a ring $R$ correspond to derivations of the inclusion $R^{q} \subset R$ (actually, we need also to assume that the Frobenius map is injective on $R$ ). In this correspondence, an $n$-derivation coming from a derivation correspond to a derivation of $R^{q} \subset R$ whose image is contained in $R^{q}$.

Fact 1.2 If $\delta: R \longrightarrow S$ is an n-derivation of $f: R \longrightarrow S$, and $I \triangleleft R, J \triangleleft S$, are ideals such that $\delta(I), f(I) \subset J$, then $\bar{\delta}: R / I \longrightarrow S / J$ is an $n$-derivation of $\bar{f}: R / I \longrightarrow S / J$. 
Proof The map $(f, \delta): R \longrightarrow S_{(1)}$ is a ring homomorphism. Let $g$ : $S \longrightarrow S / J$ be the quotient map. Then $g_{(1)}: S_{(1)} \longrightarrow(S / J)_{(1)}$ is a ring homomorphism, and $(f, \delta)(I) \subset \operatorname{ker}\left(g_{(1)}\right)=J \times J$, hence the composition:

$$
R \longrightarrow S_{(1)} \longrightarrow(S / J)_{(1)}
$$

factors through the quotient map $R \longrightarrow R / I$, inducing the homomorphism:

$$
(\bar{f}, \bar{\delta}): R / I \longrightarrow(S / J)_{(1)} \text {. }
$$

This means that $\bar{\delta}$ is an $n$-derivation of $\bar{f}$.

Fact 1.3 Suppose $R \subset R_{1}, R_{2}$ are $n$-differential ring extensions. Then there is a unique $n$-derivation on $R_{1} \otimes_{R} R_{2}$ such that the maps $R_{1}, R_{2} \rightarrow R_{1} \otimes_{R} R_{2}$ are $n$-differential. Moreover $R_{1} \otimes_{R} R_{2}$ is the coproduct of $R_{1}$ and $R_{2}$ in the natural category of $n$-differential $R$-algebras.

Proof It is a general (obvious) fact about jet operators [Kow, p. 19]. The $n$-derivation on $R_{1} \otimes_{R} R_{2}$ is given by the following formula:

$$
\delta\left(\sum_{i} a_{i} \otimes b_{i}\right)=\sum_{i} a_{i}^{q} \otimes \delta\left(b_{i}\right)+\delta\left(a_{i}\right) \otimes b_{i}^{q}
$$

Lemma 1.4 Suppose $i: R \subset S$ is an extension of domains, and $\delta: R \longrightarrow S$ is an $n$-derivation of $i$. Then $\delta$ extends to an $n$-derivation of the embedding $R_{0} \longrightarrow S_{0}$ of the fields of fractions.

Proof The map $(i, \delta): R \longrightarrow S_{(1)}$ is a ring homomorphism. Denote by $\Phi$ the following composition:

$$
R \longrightarrow S_{(1)} \longrightarrow\left(S_{0}\right)_{(1)}
$$

where the latter map comes from the embedding of $S$ into its fraction field $S_{0}$. It is enough to check, whether $\Phi$ factors through $R \longrightarrow R_{0}$. It does if $\Phi(R \backslash\{0\})$ is a subset of the multiplicative group of $\left(S_{0}\right)_{(1)}$.

Take any $r \in R \backslash\{0\}$. It is easy to check that

$$
\Phi(r) *\left(\frac{1}{i(r)},-\frac{\delta(r)}{i(r)^{2 q}}\right)=(1,0)=\mathbf{1} .
$$

Hence $\Phi(r)$ is invertible in $\left(S_{0}\right)_{(1)}$. 
Now we turn our attention to jet spaces and their connection with extensions of $\delta$. If $L$ is a field, then an affine $L$-variety is the set of zeroes in a cartesian power of $\Omega$ of a finite set of polynomials over $L$ (we do not demand $L$ irreducibility). For an $L$-variety $V$, let $I_{L}(V)=\{F \in L[\bar{X}]: F(V)=0\}$. By Hilbert Nullstellensatz and properties of polynomial ideals, $L$-varieties correspond to radical ideals in $L[\bar{X}]$, and $L$-irreducible $L$-varieties correspond to prime ideals in $L[\bar{X}]$.

For a field $L$ and a finite tuple $a \subset \Omega$, the locus of $a$ over $L$, denoted by $\operatorname{locus}_{L}(a)$, is the smallest affine $L$-variety which contains $a$. It is the set of zeroes of the ideal $I_{L}(a)=\{f \in L[\bar{X}]: f(a)=0\}$. A locus over a field $L$ is an $L$-irreducible $L$-variety and any $L$-irreducible $L$-variety is a locus over $L$ of a certain tuple $a \subset \Omega$. An affine variety $V$ is defined over $L$, if $I_{\Omega}(V)$ can be generated over $L[\bar{X}]$. Note that $\operatorname{locus}_{L}(a)$ need not be defined over $L$; e.g. if $a \in L-L^{p}$, then $\operatorname{locus}_{L^{p}}(a)$ is an $L^{p}$-variety defined over $L$ and not defined over $L^{p}$ (but still definable over $L^{p}$ ).

We fix an $n$-differential field $(K, \delta)$, and denote by $C$ the kernel of $\delta$. One checks that $C$ is a field, called the field of constants of $K$. Note some obvious consequences of the twisted Leibnitz rule $(a \in K, c \in C, m>0)$ :

$$
\delta\left(a^{m}\right)=m a^{(m-1) q} \delta(a), \quad \delta(c a)=c^{q} \delta(a)
$$

Hence, as in the case of derivations $K^{p} \subseteq C$. We call $K$ strict, if $K^{p}=C$.

If $\bar{X}=\left(X_{1}, \ldots, X_{n}\right), \bar{X}^{\prime}=\left(X_{1}^{\prime}, \ldots, X_{n}^{\prime}\right)$ are tuples of variables, then $\bar{X} \mapsto \bar{X}^{\prime}$ extends to an $n$-derivation of the inclusion $K[\bar{X}] \subset K\left[\bar{X}, \bar{X}^{\prime}\right]$ extending $\delta$ on $K$. For $F \in K[\bar{X}]$ we have a similar formula as in the (0-)differential case:

$$
\delta(F)\left(\bar{X}, \bar{X}^{\prime}\right)=F^{\delta}\left(\bar{X}^{q}\right)+\sum_{i} \frac{\partial F}{\partial X_{i}}(\bar{X})^{q} X_{i}^{\prime}
$$

where $F^{\delta}$ is the polynomial obtained from $F$ by applying $\delta$ to its coefficients, and $\bar{X}^{q}=\left(X_{1}^{q}, \ldots, X_{n}^{q}\right)$.

Definition 1.5 Let $V$ be an affine $K$-variety. The jet space of $V$, denoted by $V^{(1)}$, is the set of zeroes of $\left(I_{K}(V), \delta\left(I_{K}(V)\right)\right) \triangleleft K\left[\bar{X}, \bar{X}^{\prime}\right]$.

One can check that $V^{(1)}$ is a torsor of the $n$-th Frobenius tangent space to $V$, which is the pull-back of the tangent space to $\operatorname{Fr}^{n}(V)$ by the morphism 
$\mathrm{Fr}^{n}: V \rightarrow \mathrm{Fr}^{n}(V)$.

Also, $V \mapsto V^{(1)}$ is a functor, and we have always a projection map $V^{(1)} \rightarrow V$. For $c \in V$, we denote by $V_{c}^{(1)}$, the fibre of $V^{(1)} \rightarrow V$ over $c$.

Fact 1.6 If $a, b \in \Omega^{m}(m>0)$ and $V=\operatorname{locus}_{K}(a)$, then $(a, b) \in V^{(1)}$ if and only if $a \mapsto b$ extends to an $n$-derivation of the inclusion $K[a] \subset K[a, b]$.

Proof Obviously, $K[a] \cong K[\bar{X}] / I_{K}(a)$ and $(a, b) \in V^{(1)}$ if and only if $\delta\left(I_{K}(a)\right)$ vanishes at $(a, b)$. The latter condition is equivalent to $\delta\left(I_{K}(a)\right) \subset$ $I_{K}(a, b)$. By 1.2, $\delta\left(I_{K}(a)\right) \subset I_{K}(a, b)$ if and only if $a \mapsto b$ extends to an $n$-derivation of the inclusion $K[a] \subset K[a, b]$.

Remark Note that an $n$-derivation of $K[a] \subset K[a, b]$ not necessarily extends to an $n$-derivation of $K(a, b)$. For example we have a derivation of $K[X] \subset K\left[X^{1 / p}\right]$ induced by $\delta(X)=X^{1 / p}$, which clearly does not extend to $K\left(X^{1 / p}\right)$. In the next fact, we give conditions equivalent to the existence of an extension to the fraction field.

Fact 1.7 Let $a, b \in \Omega^{m}(m>0), V=\operatorname{locus}_{K}(a), W=\operatorname{locus}_{K}(a, b)$. The following are equivalent:

i) There exists an $n$-derivation on $K(a, b)$ extending $\delta$ and taking $a$ to $b$.

ii) There exists an $n$-differential field extension $K \subset M$ such that $a, b \subset M$ and $\delta(a)=b$.

iii) $W \subset V^{(1)}$ and $(a, b) \in \operatorname{Im}\left(W_{(a, b)}^{(1)} \rightarrow V_{a}^{(1)}\right)$.

iv) $W \subset V^{(1)}$ and $W^{\prime}:=\left\{(x, y) \in W:(x, y) \in \operatorname{Im}\left(W_{(x, y)}^{(1)} \rightarrow V_{x}^{(1)}\right)\right\}$ is Zariski dense in $W$.

\section{Proof}

i) $\longrightarrow$ ii) Obvious.

ii) $\longrightarrow$ iii) By 1.6, $(a, b) \in V^{(1)}$ and $(a, b, b, \delta(b)) \in W^{(1)}$, since $b=\delta(a)$. Therefore $W \subset V^{(1)}$, and as the map $W^{(1)} \rightarrow V^{(1)}$ is the restriction of the projection on the first and third coordinate, we get:

$$
W_{(a, b)}^{(1)} \ni(a, b, b, \delta(b)) \mapsto(a, b) \in V_{a}^{(1)} .
$$

iii) $\longrightarrow$ i) By iii), we know that the set

$$
Z:=\left\{c \in \Omega^{m}:(a, b, b, c) \in W^{(1)}\right\}
$$


is non-empty. By the form of $\delta(F)$ (for $F \in K[\bar{X}]$ ), $Z$ is also the set of zeroes of linear polynomials over $K(a, b)$, so we can find $c \in Z(K(a, b))$. By 1.6, there is an $n$-derivations of the inclusion: $K[a, b] \subset K[a, b, b, c]$ taking $a$ to $b$. By 1.4, it extends to an $n$-derivation of the inclusion $K(a, b) \subset K(a, b, b, c)$. But the latter is identity, since $c \subset K(a, b)$.

iii) is a $K$-variety. By quantifier elimination for $\mathrm{ACF}_{p}, W^{\prime}$ is $K$-constructible, therefore $(a, b) \in W^{\prime}$ if and only if $W^{\prime}$ contains a $K$-definable Zariski open subset of $W$. Since $W$ is $K$-irreducible and $W^{\prime \prime}$ is a $K$-variety containing $W^{\prime}$, the former condition is equivalent to $W^{\prime}$ being Zariski dense in $W$.

We investigate now algebraic extensions of $n$-differential fields and show that the results from differential algebra are still true in this context.

Lemma 1.8 Suppose $(K, \delta)$ is an $n$-differential field. Then $\delta$ extends in a unique way to an $n$-derivation on the separable closure of $K$. Moreover, if $K \subset L$ is any $n$-differential field extension, then the derivation on $L$ preserves the relative separable closure of $K$ in $L$.

Proof Let $b \in \Omega$ be separable over $K$, and $F \in K[X]$ be the minimal polynomial of $b$ over $K$. For $G \in K[X]$, we define $\delta$ on $K[X]$ extending the $n$-derivation on $K$ and such that $\delta(X)=G$. Then:

$$
\delta(F)(X)=F^{\delta}\left(X^{q}\right)+\left(F^{\prime q} G\right)(X)
$$

By 1.2 , we need to find a polynomial $G$ such that $I$, the ideal generated by $F$, is preserved by $\delta$. By the twisted Leibnitz rule, it is enough to find $G$ such that $\delta(F) \in I$.

Since $K[X] / I$ is a field, it is enough to show that $F^{\prime q} \notin I$. Since $F$ is separable, $F^{\prime} \neq 0$ and $\operatorname{deg}\left(F^{\prime}\right)<\operatorname{deg}(F)$. Hence $F^{\prime q}$ can not belong to the maximal ideal $I$.

Therefore, we can extend $\delta$ to $K(b)$, for any $b$ separable over $K$. Since $\mathrm{DF}_{p, n}$ is inductive, we can extend $\delta$ to the separable closure of $K$.

Uniqueness and the moreover claim follow in the same way: for $b \in L$ separable over $K$ and $F$ a minimal polynomial of $b$ over $K, \delta(b)=-\frac{F^{\delta}\left(b^{q}\right)}{F^{\prime q}(b)}$, so $\delta(b)$ is uniquely determined and separable over $K($ even $\delta(b) \in K(b))$.

In a similar manner we prove: 
Lemma 1.9 Any n-differential field has a separably closed strict extension.

Let $L_{\lambda}$ be the language of $(n$-)differential fields expanded by the symbols for the $\lambda$-functions, see [Ch, 1.8] for the definition of the $\lambda$-functions. Since the $\lambda$-functions are definable in the pure field language, any $n$-differential field has naturally the $L_{\lambda}$-structure. We will use thoroughly the fact that the extensions of fields preserving $\lambda$-functions are exactly the separable extensions [Ch, 1.9].

Fact 1.10 The theory of n-differential fields has the amalgamation property and is universal in the language $L_{\lambda}$.

Proof The proof of the amalgamation property goes similarly as in [Zi]. Let $K \subset K_{1}, K_{2}$ be extensions of $L_{\lambda}$-structures (in particular, separable extensions). Using 1.8, we can assume that $K_{1}, K_{2}$ are separably closed. Hence $K^{s}$, the separable closure of $K$ is a subfield of $K_{1}, K_{2}$. By the uniqueness and moreover claim in $1.8, K^{s}$ is an $n$-differential subfield of $K_{1}, K_{2}$ and the induced $n$-differential structures coincide. The extensions $K^{s} \subset K_{1}, K_{2}$ are still separable, because if we take a $p$-basis of $K$, it remains a $p$-basis of $K^{s}$ and is still $p$-independent in $K_{1}, K_{2}$. Therefore, we can assume (replacing $K$ by $K^{s}$ ) that the extensions $K \subset K_{1}, K_{2}$ are regular. We can also assume that $K_{1}$ is a lgebraically disjoint from $K_{2}$ over $K$. Hence, by the regularity of the extensions, $K_{1}$ is linearly disjoint from $K_{2}$ over $K$ [FJ, 9.9]. This means that $K_{1} K_{2} \cong_{K}\left(K_{1} \otimes_{K} K_{2}\right)_{0}$, the field of fractions of $K_{1} \otimes_{K} K_{2}$, and the extensions $K_{1}, K_{2} \subset K_{1} K_{2}$ are separable (even regular [FJ, 9.10]). By 1.3 and 1.4, $\delta$ extends to $K_{1} K_{2}$, so the extensions $K_{1}, K_{2} \subset K_{1} K_{2}$ are $L_{\lambda}$-extensions.

It remains to show that $\mathrm{DF}_{p, n}$ is universal in $L_{\lambda}$. The sentences expressing properties of $\lambda$-functions are actually existential, but any $L_{\lambda}$-substructure of an $L_{\lambda}$-model of $\mathrm{DF}_{p, n}$ is still an $L_{\lambda}$-model of $\mathrm{DF}_{p, n}$, since the $\lambda$-functions are also the Skolem functions for the sentences expressing $p$-independence. Namely, we can write an universal sentence expressing that a tuple is $p$ independent, as well as a universal sentence expressing that the $p$-th powers of $\lambda$-functions are $p$-coordinates of a $p$-dependent tuple.

Remark 1.11 The above fact was proved in [Kol] in the case of $n=0$ (i.e. for differential fields).

Remark 1.12 $\mathrm{DF}_{p, n}$ does not have the amalgamation property in the language of $n$-differential fields. 
Proof An example from [Wo1] works also for $n$-differential fields.

Namely, if $K$ is an $n$-differential field and $a \in K-K^{p}$ such that $\delta(a)=0$, then $\delta$ extends to $K\left(a^{1 / p}\right)$ with an arbitrary value for $\delta\left(a^{1 / p}\right)$ (since the ideal $\left(X^{p}-a\right) K[X]$ is closed under $\delta$, no matter how $\delta(X)$ is defined). Hence there are extensions of $K$ with e.g. $\delta\left(a^{1 / p}\right)=0$ and $\delta\left(a^{1 / p}\right)=1$ and they can not be amalgamated.

\section{The model companion of $\mathrm{DF}_{p, n}$}

Now we are ready to give axioms of the model companion of $\mathrm{DF}_{p, n}$, which we call $\mathrm{DCF}_{p, n}$. The axioms actually consist of a scheme of axioms.

\section{Axioms of $\mathrm{DCF}_{p, n}$}

Suppose $V, W$ are $K$-irreducible $K$-varieties, and $X$ is a proper $K$ subvariety of $W$. If $W \subset V^{(1)}, W$ projects generically onto $V$, and the set

$$
\left\{(x, y) \in W:(x, y) \in \operatorname{Im}\left(W_{(x, y)}^{(1)} \rightarrow V_{x}^{(1)}\right)\right\}
$$

is Zariski dense in $W$, then there is $a \in V(K)$ such that $(a, \delta(a)) \in$ $W-X$.

We need to say a word whether this scheme of axioms is first-order. It is rather standard (see the axiomatization of ACFA in $[\mathrm{CH}]$, or SCFA in [Ch]). The main point is that the theory $\mathrm{ACF}_{p}$ is strongly minimal, eliminates quantifiers and is complete. In particular, the most important density assumption translates as a quantifier-free formula in the language of fields about the parameters defining $W$ and $V$ saying that the Morley rank of a subset of $W$ coincides with the Morley rank of $W$. This formula is true in $\Omega$ if and only if it is true in $K$. The definability of being $K$-irreducible (even uniformly in $K$ ) is classical, see the introduction to Chapter IV in [vdD] (van den Dries also gives a new proof there).

As usual, we can remove $X$ from the assumptions by replacing $W$ with some higher-dimensional $K$-variety.

Theorem 2.1 $K$ is an existentially closed model of $\mathrm{DF}_{p, n}$ if and only if $K \models \mathrm{DCF}_{p, n}$. 


\section{Proof}

$\Longrightarrow$ Take $V, W$ and $X$ satisfying the axiom assumptions. Since $V$ and $W$ are irreducible $K$-varieties and $W$ projects generically onto $V$, there are $a, b \subset \Omega$ such that $V=\operatorname{locus}_{K}(a), W=\operatorname{locus}_{K}(a, b)$.

By the density assumption and 1.7, there exists an $n$-derivation $\delta$ on $K(a, b)$ such that $\delta(a)=b$. Obviously $(a, b) \in W-X$. Since $K$ is existentially closed, we can find $a^{\prime} \in V(K)$ such that $\left(a^{\prime}, \delta\left(a^{\prime}\right)\right) \in W-X$.

$\Longleftarrow$ By the standard trick, it is enough to show that for any quantifier-free $\zeta(x, y)$ in the language of fields, if $M=(\exists x) \zeta(x, \delta(x))$ for some $n$-differential field $M$ extending $K$, then $K \models(\exists x) \zeta(x, \delta(x))$.

Take $a \subset M$ such that $M \models \zeta(a, \delta(a))$ and let $V=\operatorname{locus}_{K}(a), W=$ $\operatorname{locus}_{K}(a, \delta(a))$. We take $X$ such that the axiom assumptions concerning it are satisfied and $\zeta(x, y)$ is implied by $(x, y) \in W-X$.

By 1.7, the density assumption holds. Hence we get $a^{\prime} \in V(K)$ such that $\left(a^{\prime}, \delta\left(a^{\prime}\right)\right) \in W-X$. Therefore $K \models(\exists x) \zeta(x, \delta(x))$.

Now, we discuss the model-theoretical properties of $\mathrm{DCF}_{p, n}$. We follow the lines from [Wo1]. Let $L$ denotes the language of $n$-differential fields and $L_{\lambda}$ the language of $n$-differential fields with $\lambda$-functions. In the next theorem we list some algebraic and model-theoretic properties of $\mathrm{DCF}_{p, n}$. We say that an $n$-differential field $K$ is $n$-differentially perfect if any $n$-differential extension of $K$ is separable.

Theorem 2.2 (i) Models of $\mathrm{DCF}_{p, n}$ (as pure fields) are separably closed and of infinite imperfection degree.

(ii) Models of $\mathrm{DCF}_{p, n}$ are $n$-differentially perfect.

(iii) $\mathrm{DCF}_{p, n}$ has the amalgamation property and the join embedding property in $L$ and $L_{\lambda}$.

(iv) $\mathrm{DCF}_{p, n}$ is the model companion of $\mathrm{DF}_{p, n}$ in $L . \mathrm{DF}_{p, n}$ does not have the model completion in $L . \mathrm{DCF}_{p, n}$ is complete.

(v) $\mathrm{DCF}_{p, n}$ is the model completion of $\mathrm{DF}_{p, n}$ in $L_{\lambda} . \mathrm{DCF}_{p, n}$ has quantifier elimination in $L_{\lambda}$.

\section{Proof}

(i) By 1.9 and 2.1, models of $\mathrm{DCF}_{p, n}$ are separably closed.

Take $K \models \mathrm{DCF}_{p, n}$ and assume that the imperfection degree of $K$ is finite. Let $a_{1}, \ldots a_{m}$ be a basis of $K$ over $K^{p}$. For convenience, we take the actual basis as opposed to a $p$-basis, so the imperfection degree of $K$ equals $\log _{p} m$. 
We are looking for a contradiction.

For $n=0$, the result was proved by Wood [Wo1]. Let us assume $n>0$.

By the universal property of the ring of polynomials, equivalence of $n$ derivations with certain homomorphisms of rings and 1.4, we can extend an $n$-derivation $\delta$ on $K$ to $K(X)$ (the field of rational functions) such that $\delta(X)=X$. Since $\mathrm{DF}_{p, n}$ is inductive, there exists $\bar{K}$, an existentially closed extension of $K$. Since $K \prec \bar{K}, a_{1}, \ldots a_{m}$ is a basis of $\bar{K}$ over $\bar{K}^{p}$. Therefore we can write

$$
X=\sum_{i=1}^{m} \alpha_{i} a_{i}
$$

for certain $\alpha_{1}, \ldots, \alpha_{m} \in \bar{K}^{p}$.

Let $B=\left(b_{i j}\right) \in M_{m}\left(K^{p}\right)$ such that for each $i \leq m, \delta\left(a_{i}\right)=\sum_{j} b_{i j} a_{j}$. Then

$$
X=\delta(X)=\sum_{i=1}^{m} \alpha_{i}^{q} \delta\left(a_{i}\right)=\sum_{i, j}^{m} \alpha_{i}^{q} b_{i j} a_{i} .
$$

Let $\bar{\alpha}=\left(\alpha_{1}, \ldots, \alpha_{m}\right)$. By the previous computation, $B \bar{\alpha}^{q}=\bar{\alpha}$.

Let $\bar{X}=\left(X_{1}, \ldots, X_{m}\right)$ be a tuple of variables and consider $B \bar{X}^{q}-\bar{X}$, the tuple of polynomials over $K$. Then $B \bar{X}^{q}-\bar{X}$ vanishes on $\alpha$ and the Jacobian of $B \bar{X}^{q}-\bar{X}$ is the identity matrix (we use $n>0$ here!).

By [La, Chapter VIII, 5.3], the extension $K \subseteq K(\bar{\alpha})$ is separable algebraic. Since $K$ is separably closed, $\alpha_{i} \in K$ for each $i \leq m$. Therefore $X \in K$, a contradiction.

(ii) Take $K \subset M$, an extension of $n$-differential fields with $K \models \mathrm{DCF}_{p, n}$. Take a finite tuple $a$ from $K$. Since $K$ is existentially closed, and the sentence expressing that $a$ is linearly dependent over the field of $p$-th powers is existential, $a$ is linearly dependent over $K^{p}$ if and only if $a$ is linearly dependent over $M^{p}$.

(iii) $\mathrm{DCF}_{p, n}$ has the amalgamation property in $L_{\lambda}$ by 1.10 and the inductiveness of $\mathrm{DF}_{p, n}$. By (ii), any $L$-extension of models of $\mathrm{DCF}_{p, n}$ is also an $L_{\lambda}$-extension, hence $\mathrm{DCF}_{p, n}$ has also the amalgamation property in $L$.

For the joint embedding property, take $K, M \models \mathrm{DCF}_{p, n}$. Let $F$ denotes the algebraic closure of the prime field. By (i), we can assume that $F$ (together with the 0 -function as the $n$-derivation) is an $n$-differential subfield of both $K$ and $M$. Since $F$ is perfect, the extensions $F \subset K, M$ are separable, hence they are $L_{\lambda}$-extensions. By 1.10 and the inductiveness of $\mathrm{DF}_{p, n}, K$ and $M$ jointly $L$-embed into a model of $\mathrm{DCF}_{p, n}$. $\mathrm{By}$ (ii), $\mathrm{DCF}_{p, n}$ has also the joint 
embedding property in $L_{\lambda}$.

(iv) Since $\mathrm{DF}_{p, n}$ is inductive, the model companion of $\mathrm{DF}_{p, n}$ exists if and only if the class of existentially closed models of $\mathrm{DF}_{p, n}$ is elementary, and is given by an axiomatization of this class [Ho, 8.3.6].

By (iii) and 2.1, $\mathrm{DCF}_{p, n}$ is complete.

Since $\mathrm{DF}_{p, n}$ does not have the amalgamation property in $L$ (1.12), it does not have the model completion in $L$.

(v) By (ii), any $L$-extension of a model of $\mathrm{DCF}_{p, n}$ is also an $L_{\lambda}$-extension, hence (by (iii)) $\mathrm{DCF}_{p, n}$ is the model companion of $\mathrm{DF}_{p, n}$ in $L_{\lambda}$ as well. Since $\mathrm{DF}_{p, n}$ has the amalgamation property and is universal in $L_{\lambda}(1.10), \mathrm{DCF}_{p, n}$ is the model completion of $\mathrm{DF}_{p, n}$ in $L_{\lambda}$ and $\mathrm{DCF}_{p, n}$ has quantifier elimination in $L_{\lambda}$.

In the theory $\mathrm{DCF}_{p}$, we get the elimination of quantifiers just after adding the $\lambda_{0}$-function (the inverse of Frobenius on $K^{p}$, zero elsewhere). It is implied by the fact that the strict differential fields are differentially perfect. We do not know, if this is true in the case of $n$-differential fields, however (as in [Wo1]), if a field is $n$-differentially perfect, then it is strict (see the proof of $1.12)$.

What we really need is the property that for any extension $K \subset L$ of $n$ differential fields, the constants of $L$ are linearly disjoint from $K$ over the constants of $K$. To prove this the Wronskian technique is needed. This technique does not work in the $n$-differential case (for $n>0$ ). For instance the solutions of the equation $X^{\prime \prime}=0$ can have infinite dimension over constants: e.g. if $\delta=F r^{n} \circ \delta^{\prime}(n>0)$, where $\delta^{\prime}$ is a derivation on $K$ such that $K$ has the infinite dimension over constants of $\delta^{\prime}$. The usage of Wronskian for $n=0$ gives that the dimension of the solution set of the equation $X^{\prime \prime}=0$ is not bigger than 2 .

Using Wronskian, one can also easily show that models of $\mathrm{DCF}_{p}$ have infinite imperfection degree: dependence over constants becomes a quantifier-free condition, so having big imperfection degree becomes an existential condition. Note also that our proof of 2.1(i) does not work in the (0-)differential case.

The reason of the non-existence of Wronskian for $n>0$ is the following: the composition of two $n$-derivations is a $2 n$-derivation, and to use Wronskian effectively we need higher order operators. But maybe some other techniques may be used, or the higher order operators may be obtained in an indirect 
way, so we find it natural to ask the following:

Question 1 Let $K \subset L$ be an $n$-differential field extension. Are the constants of $L$ linearly disjoint from $K$ over the constants of $K$ ?

Question 2 Is any strict $n$-differential field $n$-differentially perfect?

Question 3 Does $\mathrm{DCF}_{p, n}$ has elimination of quantifiers in the language with the inverse of Frobenius?

As it was explained above, the positive answer to Question 1 implies the positive answer to Question 2 and the positive answer to Question 2 implies the positive answer to Question 3.

Our axioms of $\mathrm{DCF}_{p, n}$ include the Wood axiomatization [Wo1] of $\mathrm{DCF}_{p}$ in the case of $n=0$. Let us recall these axioms:

- $K$ is strict.

- If $F, G$ are differential polynomials over $K$ in one variable, $\operatorname{ord}(G)=$ $m$, ord $(F)<m$, and $\frac{\partial G}{\partial X^{(m)}} \neq 0$, then there exists $a \in K$ such that $G(a)=0, F(a) \neq 0$.

Note that these axioms make perfect sense also for $n$-derivations.

If we set $V=A^{m}, W=Z\left(X_{1}^{\prime}=X_{2}, \ldots, X_{m-1}^{\prime}=X_{m}, G\left(X_{1}, \ldots, X_{m}, X_{m}^{\prime}\right)\right)$, $X=W \cap Z\left(F\left(X_{1}, \ldots, X_{m}\right)\right)$, then finding $a \in K$ such that $G(a)=0, F(a) \neq 0$ is equivalent to finding $a \in V(K)$ such that $(a, \delta(a)) \in W-X$ (for $\delta$, an $n$ derivation on $K)$. The condition $\frac{\partial G}{\partial X^{(m)}} \neq 0$ means exactly that the morphism $W \rightarrow V$ is separable. This morphism is separable if and only if the tangent morphism $T_{w} W \rightarrow T_{v} V$ is surjective for a generic $w \in W$ if and only if the $n$ th Frobenius tangent morphism $F^{n} T_{w} W \rightarrow F^{n} T_{v} V$ is surjective for a generic $w \in W$. As the jet space is a torsor of the $n$-th Frobenius tangent space (see remark after 1.5), the separability of $W \rightarrow V$ implies the density assumption axioms of $\mathrm{DCF}_{p, n}$. So the Wood axioms are a part of our axioms in the case of $n=0$, similarly as in the characteristic zero case, the Blum axioms are a part of Pierce-Pillay axioms $[\mathrm{PP}]$. For $p=0$, the density assumption is superfluous, since a dominant morphism is always separable. Removing this assumption from our axioms, we get exactly the Pierce-Pillay axioms of $\mathrm{DCF}_{0}$. Pierce $[\mathrm{Pi}]$ has recently given new geometric axioms of DCF (including the positive characteristic case) and ACFA of somewhat dual nature to the 
original axioms.

It is natural to ask the following:

Question 4 Suppose that the density assumption in Axiom 2 is replaced by the separability of the morphism $W \rightarrow V$ together with the strictness of $K$. Does it still axiomatize $\mathrm{DCF}_{p, n}$ ?

Question 5 Can $\mathrm{DCF}_{p, n}$ be axiomatized by the Wood axioms?

The positive answer to Question 5 implies the positive answer to Question 4 , since the axioms from Question 4 still include the Wood axioms.

The last question concerns the behavior of compositions of $n$-derivations:

Question 6 Suppose $n>0$ and $(K, \delta)$ is a model of $\mathrm{DCF}_{p, n}$. Is $(K, \delta \circ \delta) a$ model of $\mathrm{DCF}_{p, 2 n}$ ?

To prove stability of $\mathrm{DCF}_{p, n}$ we need the fact below. For necessary definitions see [De2]. We say that for $K \subset L \subset F, K \subset M \subset F$, separable extensions of fields, $L$ is $p$-disjoint from $M$ over $K$ in $F$, if any subset of $L$ which is $p$-free over $K$ in $F$, remains $p$-free over $M$ in $F$. This is equivalent to saying that there exist $p$-bases $B_{K}, B_{L}, B_{M}$ of $K, L, M$ respectively such that $B_{K} \subset B_{L}, B_{M}$ and $B_{L} \cup B_{M}$ is $p$-independent in $F$. By [De1, p. 63], if $F$ above is an enough saturated model of $\mathrm{SCF}_{p, \infty}$ (the theory of separably closed fields of infinite imperfection degree), then $L$ is forking independent from $M$ over $K$ if and only if $L$ is $p$-disjoint and algebraically disjoint (in this case equivalently linearly disjoint) from $M$ over $K$ in $F$.

Fact 2.3 Suppose $K \subset L \subset F, K \subset M \subset F$ are separable extensions of $n$ differential fields, $F$ (as a pure field) is an enough saturated model of $\mathrm{SCF}_{p, \infty}$ and $a \in F$. Then:

i) If $L$ is p-disjoint from $M$ over $K$ in $F$, then the extension $L M \subset F$ is separable.

ii) There exists $K_{a}$, a countable $L_{\lambda}$-substructure of $F$ containing a, such that $K_{a}$ is $\mathrm{SCF}_{p, \infty}$-independent from $K$ over $K_{a} \cap K$.

\section{Proof}

i) This is a fact about pure separable fields, and its proof appears in [Wo2].

It is enough to notice that $B_{L} \cup B_{M}\left(B_{L}, B_{M}\right.$ as above) is a $p$-basis of $L M$ 
and is still $p$-independent in $F$.

ii) By stability of $\mathrm{SCF}_{p, \infty}$, for any countable $a \subset F$, there exists a countable field $k \subset K$ such that $a$ is $\mathrm{SCF}_{p, \infty}$-independent from $K$ over $k$. Then $K_{(a)}$, the smallest $\lambda$-closed subfield of $F$ containing $k(a)$ satisfies the statement of (ii) except it is not $n$-differential subfield. But we can take $a_{1}$, the $n$ differential field generated by $K_{(a)}$, and apply to this countable tuple the above construction again. Then we finish using the usual chain procedure.

Theorem 2.4 $\mathrm{DCF}_{p, n}$ is stable and not superstable.

Proof Since models of $\mathrm{DCF}_{p, n}$ are not perfect (as fields), $\mathrm{DCF}_{p, n}$ is not superstable. To prove stability we follow the Shelah's proof of stability of $\mathrm{DCF}_{p}[\mathrm{Sh}]$. We need to prove that for any pair $K \subset L$ of models of $\mathrm{DCF}_{p, n}$, there at most $|K|^{\aleph_{0}}$ 1-types over $K$ of elements of $L$. We can assume that $L$ (as a pure field) is an enough saturated model of $\mathrm{SCF}_{p, \infty}$. By 2.5(ii), to any $a \in L$ we can associate $K_{a}$, a countable $L_{\lambda}$-substructure of $L$ such that $a \in K_{a}$ and $K_{a}$ is linearly disjoint and $p$-independent from $K$ over $K \cap K_{a}$.

For $a, b \in L$, we let $a \sim b$, if $K \cap K_{a}=K \cap K_{b}$ and $K_{a}$ is $n$-differentially isomorphic to $K_{b}$ over $K \cap K_{a}$ by a map sending $a$ to $b$. There are obviously $\leq|K|^{\aleph_{0}}$ equivalence classes of $\sim$. Suppose $a \sim b$ and let $k:=K \cap K_{a}$. By 1.3, we can extend the $n$-differential isomorphism between $K_{a}$ and $K_{b}$ to an $n$ differential isomorphism $K_{a} \otimes_{k} K \cong_{K} K_{b} \otimes_{k} K$. By the linear disjointness, we get a field isomorphism $\left(K_{a} \otimes_{k} K\right)_{0} \cong_{K} K_{a} K$ and $\left(K_{b} \otimes_{k} K\right)_{0} \cong_{K} K_{b} K$. These isomorphisms are $n$-differential by the uniqueness of the $n$-derivations on the fields in question. Hence $K_{a} K$ and $K_{b} K$ are $n$-differentially isomorphic over $K$. The extensions $K K_{a}, K K_{b} \subset L$ are separable by 2.5 (i), so $K K_{a}, K K_{b}$ are $L_{\lambda}$-substructures of $L$. By 2.4, $\mathrm{DCF}_{p, n}$ has elimination of quantifiers in $L_{\lambda}$, thus $a$ and $b$ have the same types over $K$.

Therefore, the equality of types is coarser than $\sim$, so there are $\leq|K|^{\aleph_{0}}$ types over $K$.

The proof of 2.6 also shows that the non-forking independence relation in $\mathrm{DCF}_{p, n}$ is (as usual) the natural one: $A$ is independent from $B$ over $C$, if $<A>_{\lambda}$ is $\mathrm{SCF}_{p, \infty}$-independent from $<B>_{\lambda}$ over $<C>_{\lambda}$, where for a set $D,<D>_{\lambda}$ is the $L_{\lambda}$-substructure generated by $D$.

The theory $\mathrm{DCF}_{p, n}$ is an expansion of $\mathrm{SCF}_{p, \infty}$, which behaves to a certain degree similarly as $\mathrm{DCF}_{p}$. It seems interesting to go to the limit with $n$, i.e. 
to consider the theory $\mathrm{DCF}_{p, \infty}$, the limit theory (ultraproduct) of $\mathrm{DCF}_{p, n}$ as $n$ goes to infinity. This will be done in the forthcoming paper.

\section{References}

[Bu] A. Buium, Arithmetic Analogues of Derivations, Journal of Algebra 198 (1997), 290-299.

[Ch] Z. Chatzidakis, Generic automorphisms of separably closed fields, Illinois Journal of Mathematics 45 Nr 3 (2002), 693-733.

[CH] Z. Chatzidakis and E. Hrushovski, Model theory of difference fields, Transactions of AMS $3 \mathbf{5 1}$ (1999), 2997-3071.

[De1] F. Delon, Idéaux et types sur les corps séparablement clos, Supplément au Bulletin de la SMF, Mémoire 33, Tome 116 (1988).

[De2] F. Delon, Separably closed fields in Model Theory and Algebraic Geometry, E. Bouscaren (Ed.), Springer-Verlag (1987).

[FJ] M. D. Fried, M. Jarden Field Arithmetic, Springer-Verlag (1986).

[Ho] W. Hodges, Model Theory, Cambridge University Press (1993).

[Kol] E. R. Kolchin, Differential Algebra and Algebraic Groups, Academic Press, New York (1973).

[Kow] P. Kowalski, Some model-theoretical and geometric properties of fields with jet operators, Ph.D. thesis, Wrocław University (2001).

[La] S. Lang, Algebra, Springer-Verlag (2002).

[Pi] D. Pierce, Geometric characterization of existentially closed fields with operators, preprint.

http://www.math.metu.edu.tr/ dpierce/papers/gmc/gmc.dvi

[PP] D. Pierce and A. Pillay, A Note on the Axioms for Differentially Closed Fields of Characteristic Zero, Journal of Algebra 204 (1998), 108-115. 
[Sc] T. Scanlon, Model Theory of Valued D-fields with Applications to Diophantine Approximations in Algebraic Groups, Ph.D. thesis, Harvard University (1997).

http://math.berkeley.edu/ scanlon/papers/thesis.ps

[Sh] S. Shelah, Differentially closed fields, Israel Journal of Mathematics 16 (1973), 314-328.

[Wo1] C. Wood, The model theory of differential fields revisited, Israel Journal of Mathematics 25 (1976), 331-352.

[Wo2] C. Wood, Notes on the separability of separably closed fields, Journal of Symbolic Logic 44 no. 3 (1979), 412-416.

[vdD] L. van den Dries, Decidability and bounds for polynomial ideals, Ph.D. thesis, Utrecht University (1978).

[Zi] M. Ziegler, Separably closed fields with Hasse derivations, Journal of Symbolic Logic 68 (December 2003), 311-318. 